\title{
"Que sor la raïne versa /Tot lo vin de coi [la cope] estoit plaine ». L'injure faite à la reine dans quelques textes narratifs du Moyen Âge central
}

\section{Lisa Sancho}

\section{(2) OpenEdition}

Journals

Édition électronique

URL : http://journals.openedition.org/questes/5610

DOI : 10.4000/questes.5610

ISSN : 2109-9472

Éditeur

Les Amis de Questes

\section{Édition imprimée}

Date de publication : 11 novembre 2019

Pagination : 61-81

ISSN : 2102-7188

\section{Référence électronique}

Lisa Sancho, « "Que sor la raïne versa /Tot lo vin de coi [la cope] estoit plaine ». L'injure faite à la reine dans quelques textes narratifs du Moyen Âge central », Questes [En ligne], 41 | 2019, mis en ligne le 20 décembre 2019, consulté le 20 février 2020. URL : http://journals.openedition.org/questes/5610 ; DOI : 10.4000/questes.5610 


\title{
«Que sor la raïne versa /Tot lo vin de coi [la cope] estoit plaine ». L'injure faite à la reine dans quelques textes narratifs du Moyen Âge central
}

\author{
Lisa SANCHO \\ Université Bourgogne Franche-Comté - CPTC
}

Et la reine qui est con comme une chaise ${ }^{1}$ !

Si l'injure portée contre le roi au Moyen Âge a déjà fait l'objet d'un certain nombre de travaux de manière directe ou oblique ${ }^{2}$, celle faite à la reine n'a pas suscité, jusqu'à maintenant, un intérêt aussi prononcé ${ }^{3}$. Ce cas spécifique d'offense se situe pourtant au carrefour de deux enjeux qui mériteraient sans doute que l'on s'y attarde davantage : l'affront infligé à une souveraine cible en effet à la fois un genre, ici le féminin, et un statut de rang supérieur, en l'occurrence royal. La première perspective implique que la physionomie de l'outrage souillant l'honneur

\footnotetext{
${ }^{1}$ Kaamelott, série télévisée créée par Alexandre Astier, Alain Kappauf et Jean-Yves Robin, CALT, Livre II, épisode 37, « L’Escorte II », 2005.

${ }^{2}$ Parmi ces études, citons simplement Jacqueline Hoareau-Dodineau, Dieu et le roi. La répression du blasphème et de l'injure au roi à la fin du Moyen Âge, Limoges, PULIM, coll. «Cahiers de 1'Institut d'anthropologie juridique », 2002 ; Bénédicte Sère, «Le roi peut-il avoir honte ? Quelques réflexions à partir des chroniques de France et d'Angleterre (XII ${ }^{\mathrm{e}}-\mathrm{XIII}^{\mathrm{e}}$ siècle)», dans Politiques des émotions au Moyen Âge, dir. Damien Boquet et Piroska Nagy, Florence, Sismel - Edizioni del Galluzzo, coll. « Micrologus' Library », 2010, p. 49-74.

${ }^{3}$ Signalons néanmoins le très bel ouvrage de Yasmina Foehr-Janssens, qui aborde le problème sous l'angle du motif de la femme persécutée : La Veuve en majesté. Deuil et savoir au féminin dans la littérature médiévale, Genève, Droz, coll. «Publications romanes et françaises », 2000.
} 
d'un personnage féminin revêt des spécificités propres; la seconde, qu'une telle avanie s'inscrit dans un cadre plus large, au sein duquel l'humiliation subie par la figure réginale menace de rejaillir, par extension, sur l'image du roi puis d'affecter, par analogie, l'équilibre de la royauté ${ }^{4}$.

La présente étude vise donc à montrer que, par des ressorts mettant en jeu la sémantique et la pragmatique du discours comme du geste, l'injure faite à la reine invite à une réflexion sur les forces et les faiblesses du pouvoir tel qu'il est représenté et pensé dans quelques œuvres de la littérature narrative du XII ${ }^{\mathrm{e}}$ et de l'aube du XIII ${ }^{\mathrm{e}}$ siècle $^{5}$.

\section{Injurier la reine : de la figure réginale au couple royal}

Analysées en fonction de leur nature et de leur orientation genrée, les manifestations de l'offense dirigée contre la reine littéraire permettent de mesurer combien, dans les textes retenus ici, la figure réginale est construite non pas en tant que personnage autonome, mais toujours en relation de dépendance vis-à-vis de son royal époux.

\footnotetext{
${ }^{4}$ Il convient de signaler dès à présent la dualité politique et physique qui sous-tend la conception du pouvoir monarchique à partir $\mathrm{du} \mathrm{XI}$ siècle et préfigure la théorie des « deux corps du roi » (laquelle s'épanouit à partir de la fin du Moyen Âge, surtout en Angleterre) : on distingue en effet la royauté, sacrée et permanente, et la personne individuelle incarnant provisoirement ce statut royal. Pour plus de précisions, voir Ernst Kantorowicz, Les Deux Corps du roi. Essai sur la théologie politique au Moyen Âge [1957], trad. Jean-Philippe et Nicole Genet, Paris, Gallimard, 1989.

${ }^{5}$ Bien qu'ils revêtent un intérêt incontestable dans le cadre d'une réflexion sur l'injure faite à la reine, nous choisissons d'exclure du corpus certains textes : l'épisode d'Iseut livrée aux lépreux dans Le Roman de Tristan de Béroul, celui de la Fausse Guenièvre dans le Lancelot en prose, celui où cette même souveraine est conduite au bûcher dans La Mort le roi Artu, ou encore les malheurs de l'héroïne éponyme dans la Berte aus grans piés d'Adenet le Roi. En effet, tous ces passages ont en commun de mettre en scène des situations où la reine est bafouée non par un quelconque tiers, mais par son propre époux. Le contexte initial de l'injure étant modifié, les conséquences s'en trouvent elles aussi changées.
} 
L'injure verbale et gestuelle : entre topos misogyne de la luxure féminine et violence exacerbée

Les attaques verbales et gestuelles sont sans nul doute les plus fréquentes au sein du corpus, et il arrive souvent qu'elles soient combinées entre elles. Une telle association est particulièrement patente dans la scène de Laon qui apparaît dans la chanson de geste Aliscans. Après le désastre de Larchamp, où toute l'armée du comte Guillaume d'Orange a été décimée par les Sarrasins, celui-ci se rend à la cour pour prier son beau-frère le roi Louis, fils de Charlemagne, d'engager les troupes royales dans la guerre. Après un refus initial, le souverain accepte finalement de l'aider, terrifié par l'accès de rage de son vassal. Mais la reine Blanchefleur, épouse de Louis et sœur de Guillaume, intervient afin de s'opposer à cette alliance pour des raisons égoïstes. Le héros se lance alors dans une diatribe ordurière et infamante :

«Tes toi », dist il, «pute lisse provee. /Tiebaut d'Arrabe vos a ensoinantee/Et meinte foiz comme putein folee, $/ \mathrm{Ne}$ doit pas estre ta parole escoutee. [...]/Pute mauvese, vil lisse abandonnee, /Mout avez hui ma parole blasmee /Et vers le roi m'aïe destornee./Li vif deable vos ont or coronee. ${ }^{6} \gg$

Les nombreuses injures verbales proférées ici montrent que les accusations portées par le frère contre la sœur se polarisent sur le domaine de la sexualité : les très explicites références à la débauche supposée de Blanchefleur, ainsi que l'anaphore de l'insulte «pute »/«putein », font clairement signe du côté du péché de luxure. La dynamique à l'œuvre dans cette invective révèle un dérèglement

\footnotetext{
6 Aliscans, éd. Claude Régnier, Paris, Honoré Champion, coll. «Champion Classiques », 2007, LXIX, p. 238-240, v. 3190-3208. Ce passage constitue en fait une réécriture très proche, sur le plan de l'argumentation et du vocabulaire, du même épisode déjà présenté dans La Chanson de Guillaume, éd. et trad. François Suard, Paris, Librairie Générale Française, coll. « Lettres gothiques », 1996, v. 2603-2617.
} 
fonctionnel $^{7}$. En dressant de sa sœur un portrait de femme lascive et dépravée, topos misogyne largement inscrit dans les mentalités médiévales, Guillaume lui dénie le droit d'incarner la première fonction dumézilienne et la cantonne dans la troisième, qu'il dégrade au passage en la présentant à travers le prisme du dévergondage. Ce faisant, le comte rabaisse sa suzeraine au rang de femme tout à fait ordinaire.

La suite de l'épisode prolonge ce mouvement, cette fois en avilissant physiquement le personnage réginal. Devant la cour assemblée, le frère arrache la couronne de la tête de sa sœur et la jette à terre, avant de saisir Blanchefleur par les cheveux. Il empoigne ensuite son épée et semble prêt à la décapiter lorsque Hermenjart, nulle autre que la mère du comte et de la reine, empêche in extremis le drame de survenir. L'épouse de Louis offre alors un spectacle pitoyable: honteuse, échevelée, semblable à une folle, elle fuit la scène de son humiliation pour se réfugier dans sa chambre où, de peur, elle tombe évanouie ${ }^{8}$. La tête de la reine se voit donc profanée par trois fois en l'espace de quelques vers : d'abord avec la couronne jetée à terre, puis avec la coiffure défaite et jusqu'au paroxysme de la quasi-décapitation. Or, en raison de l'importance symbolique conférée au chef et, par extension, au couvrechef, qui signalent le statut social et la dignité morale de l'individu, cette triple atteinte - capitale dans tous les sens du terme - constitue un très

\footnotetext{
${ }^{7}$ Rappelons que Georges Dumézil a mis en évidence, chez les peuples indoeuropéens, l'existence d'une tripartition des structures communes à un ordre du monde, appelées «fonctions ». La première fonction touche au sacré, c'est-à-dire au rapport des hommes avec le divin et, notamment, à l'intermédiaire que constitue le pouvoir exercé par le roi ; la deuxième concerne essentiellement le domaine militaire ; enfin, la troisième relève des notions d'abondance, de production, et inclut en particulier le champ de la sexualité. À la fois personnage au statut sacré et au rôle reproducteur, la reine s'inscrit normalement aussi bien dans la première fonction que dans la troisième. Voir Georges Dumézil, L'Idéologie tripartite des Indo-Européens, Bruxelles, Latomus, 1958.

${ }^{8}$ Aliscans, éd. cit., LXIX, p. 240, v. 3209-3220.
} 
grave coup porté à l'honneur de la souveraine, et même un crime de lèse-majesté ${ }^{9}$.

En joignant le geste à la parole, le comte d'Orange illustre bien ce qui fait la spécificité de l'outrage infligé à un personnage féminin. Fondé sur le ressort misogyne de la lubricité, ce type d'offense se caractérise surtout par une violence prononcée. Si le roi peut lui aussi être injurié, parfois même de manière virulente, il ne l'est jamais, en revanche, avec ce degré de brutalité. C'est parce que Blanchefleur est d'abord femme avant d'être reine que Guillaume s'emporte tant.

\section{Injurier la reine, injurier le roi : un phénomène de capillarité}

La négation du statut sacré de la reine, qui l'abaisse au rang de femme ordinaire, constitue un blasphème, d'autant qu'un lien indéfectible unit la figure réginale à celle du roi. Cette relation de dépendance asymétrique, où la reine est subordonnée à son époux, crée un mouvement spéculaire systématique : tout ce qui affecte le membre féminin du couple royal rejaillit de facto sur son autre moitié, masculine.

Dans l'incipit du Conte du Graal de Chrétien de Troyes, le héros Perceval, tout juste arrivé à la cour d'Arthur, est surpris devant l'attitude singulière de ce roi censé « faire les chevaliers » et qui, pourtant, apparaît abîmé dans ses pensées. Ce repli mélancolique d'Arthur se justifie par le fait que le Chevalier Vermeil vient de revendiquer la terre de Logres pour lui-même. Surtout, comme le souverain l'explique à Perceval :

«Et la raïne devant moi/Estoit ci venue seoir/Por conforter et por veoir/Ces chevaliers qui sont blecié. / $\mathrm{Ne}$ m'aüst guaires correcié $/ \mathrm{Li}$ chevaliers de quant qu'il dit, /Mais devant moi ma cope prist /Et si folement l'an leva/Que sor la raïne versa /Tot lo vin de coi estoit plaine. /Ci

\footnotetext{
${ }^{9}$ Nicole Gonthier, "Sanglant Coupaul!", "Orde Ribaude! ». Les injures au Moyen Âge, Rennes, Presses universitaires de Rennes, coll. « Histoire », 2007, p. 16.
} 
ot oevre laide et vilaine, /Que la raïne en est entree, /De dol et d'ire enflamee, /En sa chambre, ou ele s'ocit, $/ \mathrm{Ne}$ ne cuit pas, que Dex m'aït, /Que ja en puisse eschaper vive. ${ }^{10}$ "

Ainsi, par la souillure concrète résultant du renversement de la coupe remplie de vin sur Guenièvre, l'outrage gestuel du Chevalier Vermeil à l'encontre de la reine est vécu comme une humiliation plus grande que celle infligée à Arthur. La peinture des émotions de la souveraine ainsi que sa fuite, qui rappellent ce que l'on a pu lire au sujet de Blanchefleur, achèvent de souligner la gravité du crime perpétré. Or, en vertu du lien unissant étroitement la reine à son époux, Arthur est victime d'une double offense, et donc d'une double humiliation: non seulement il se voit directement provoqué par le Chevalier Vermeil qui lui réclame sa terre, mais il subit également par capillarité l'affront infligé à son épouse. Significativement, c'est d'ailleurs lui qui livre à Perceval le récit de l'outrage commis contre Guenièvre, ce qui prouve combien il se l'est approprié et combien il en est affecté. Au fond, une telle identification avec une injure initialement dirigée contre sa femme, et non contre lui, n'est pas sans fondement: en la souillant du vin de sa propre coupe, l'intention du Chevalier Vermeil est sans doute moins d'humilier la reine elle-même que de viser, à travers elle, le roi Arthur ${ }^{11}$.

\footnotetext{
${ }^{10}$ Chrétien de Troyes, Le Conte du Graal, éd. Charles Méla, Paris, Librairie Générale Française, coll. « Lettres gothiques », 1990, p. 86-88, v. 910-925.

${ }^{11}$ Cette observation peut également être appliquée à Aliscans et invite à examiner, sous un jour nouveau, l'acharnement du comte d'Orange contre Blanchefleur. Certes, la réaction égoïste de cette dernière à sa requête militaire, ainsi que le tempérament sanguin du héros, justifient en bonne partie la fureur guillelmienne. Mais cet emportement excessif tient peut-être aussi à une raison politique: ne pouvant vitupérer contre le roi avec la même violence, au motif que ce dernier est l'incarnation suprême de la royauté, le comte d'Orange reporte toute son offensive sur l'épouse de celui-ci, et le fait d'autant plus impunément qu'elle est sa sœur. L'injure à la reine fonctionne ainsi comme le vecteur de la frustration du vassal qui, au lieu de s'en prendre ouvertement à son suzerain, évacue sa colère en dirigeant ses insultes contre la figure réginale (sur les griefs du héros, voir le développement consacré au «Portrait du roi en souverain pusillanime »). Preuve, si besoin était, que la reine littéraire a beau
} 
Par conséquent, loin de se limiter à la seule personne de la souveraine, les injures perpétrées contre elle ont des répercussions qui s'étendent jusqu'à son royal époux. Injurier la reine équivaut donc, par capillarité, à injurier le roi. Plus encore, l'avanie infligée à la reine vise finalement moins, dans son intention, le membre féminin du couple royal que sa moitié masculine : en attaquant son épouse, c'est toujours le roi que l'on cherche à atteindre.

\section{L'injure faite à la reine comme agent révélateur des défaillances du couple royal}

Si l'outrage dirigé contre la figure réginale touche par capillarité le monarque, il semble légitime de supposer que, d'un point de vue diégétique, une telle offense puisse être considérée comme un élément perturbateur susceptible de faire vaciller le pouvoir incarné par le binôme royal. L'étude du corpus révèle néanmoins que les dynamiques narratives à l'œuvre diffèrent de ce à quoi l'on aurait pu s'attendre.

\section{Portrait du roi en souverain pusillanime}

Dans Aliscans, juste avant de s'emporter contre Blanchefleur, Guillaume s'en prend de manière assez virulente à son beau-frère qui, rappelons-le, a initialement refusé de le soutenir militairement :

«Looïs sire, ci a mal soudee. /Quant a Paris fu la cort assemblee, /Que Charlemaine ot vie trespassee, /Vil te tenoient tuit cil de la contree. /De toi fust France tote desheritee, /Ja

être intrinsèquement liée au roi, elle n'est pas pour autant son alter ego, mais lui est subordonnée. Le constat vaut d'ailleurs également sur le plan historique : si la reine de France s'illustre bien en tant que consors regni jusqu'au XII ${ }^{\mathrm{e}}$ siècle approximativement, elle est ensuite globalement écartée de l'exercice du pouvoir. Voir Régine Le Jan, Femmes, pouvoir et société dans le haut Moyen Âge, Paris, Picard, 2001, ainsi que Murielle Gaude-Ferragu, La Reine au Moyen Âge. Le pouvoir féminin $\left(X I V^{e}-X V^{e}\right.$ siècle), Paris, Tallandier, 2014. 
la corone ne te fust otroiee, /Quant j'en sofri por toi si grant mellee/Que maugré aus fu en ton chief posee /La grant corone qui d'or est esmeree. ${ }^{12} »$

$\mathrm{Au}$ cœur des accusations portées par le comte d'Orange contre son suzerain, figure un rappel de faits antérieurs qui se sont déroulés dans $L e$ Couronnement de Louis. Le jeune monarque, «coart eritier ${ }^{13}$ » selon les termes de son propre père Charlemagne, voit son droit à lui succéder menacé d'emblée par les ambitions de quelques barons. Il ne doit finalement sa couronne qu'à Guillaume qui, en position de supériorité physique et politique, procède lui-même à l'intronisation et se charge ensuite de réduire à néant les tentatives d'usurpation du trône. Si le pouvoir revient donc bien à l'héritier légitime, c'est ici moins le droit que la force qui fait loi.

Pourquoi une telle analepse à ce moment du récit ? Il s'agit en fait, en prolongeant dans Aliscans le portrait ébauché de Louis au sein du Couronnement, de mettre en évidence la pusillanimité du souverain ancrée en lui depuis le tout début de son règne. À travers l'illustration de cette faiblesse, les deux chansons pointent son inaptitude à assumer sa fonction sacrée. La charge verbale de Guillaume à l'encontre de son suzerain, redoublée par la violence de son geste envers Blanchefleur, résonne alors comme un écho inversé au couronnement de Louis par le comte d'Orange. Après avoir autoritairement adjugé le pouvoir monarchique au fils de Charlemagne et, par extension, à son épouse, le héros procède ici à une destitution symbolique. Sanctionnant leur ingratitude, Guillaume déchoit virtuellement de leur royauté Louis, en vilipendant sa faiblesse, et Blanchefleur, en la décoiffant dans tous les

\footnotetext{
${ }^{12}$ Aliscans, éd. cit., LXIX, p. 238, v. 3171-3179.

${ }^{13}$ Le Couronnement de Louis, éd. Ernest Langlois, Paris, Honoré Champion, coll. « Classiques français du Moyen Âge », 2013, IX, p. 4, v. 92.
} 
sens du terme, leur ôtant ainsi l'autorité régalienne qu'il leur avait luimême conférée.

On perçoit donc bien que ce n'est pas l'outrage commis par Guillaume qui fragilise le pouvoir royal : c'est au contraire parce que celui-ci est déjà affaibli par les manquements du couple qui l'incarne que le vassal s'autorise à rappeler à son suzerain qu'il lui doit sa couronne. $\mathrm{Au}$ fond, l'injure opère ici bien plus comme un agent révélateur des insuffisances d'une autorité déjà vacillante dans ses fondations que comme un facteur déclenchant.

\section{L'injure imaginaire ou le sombre visage d'une male reine}

Au-delà de la seule faiblesse, le binôme royal peut être gangrené par des vices plus pernicieux. Ainsi, dans le lai de Lanval de Marie de France, la reine est éprise du personnage éponyme, chevalier de son époux le roi Arthur, et lui fait connaître très directement les élans de son cœur. Le héros repousse ses avances, arguant de sa loyauté envers son souverain ; elle, de son côté, prend ce refus comme un outrage. L'affront est ici imaginaire : le comportement de Lanval lui est officiellement dicté par le lien vassalique qui l'unit à son suzerain. En refusant de devenir l'amant de la reine, le jeune chevalier fait montre d'une fidélité qui l'honore. À l'inverse, la réaction de la souveraine est indigne de son rang :

La reïne s'en curuça;/Iriee fu, si mesparla. / Lanval, fet ele, bien le quit, /Vus n'amez gueres cel deduit. /Asez le m'ad hum dit sovent, /Que des femmes n'avez talent !/Vallez avez bien afeitiez, /Ensemble od eus vus deduiez. /Vileins cüarz, mauveis failliz, /Mut est 
mis sire maubailliz, $/ \mathrm{Ki}$ pres de lui vus ad suffert, /Mun escïent que Deu en pert. ${ }^{14} \gg$.

La colère de l'épouse d'Arthur est à la source d'une injure, réelle cette fois, envers Lanval. Le lien de cause à effet est d'ailleurs mis en relief par la construction syntaxique des deux premiers vers de la citation, où l'adverbe à valeur consécutive «si » traduit bien le passage du courroux («s'en curuça ») et de la fureur («[i]riee ») à l'acte de « mesparl[er]». Les insultes «[v]ileins cüarz » et « mauveis failliz » visent à stigmatiser, par l'inversion sexuelle, un supposé manque de virilité du jeune homme ${ }^{15}$ et à justifier, par analogie abusive, la fausse accusation d'homosexualité. Autrement dit, l'infamia imaginaire attribuée à Lanval entraîne une infamia réelle perpétrée par la reine contre le chevalier, afin de propager sa mala fama. L'épisode souligne en fait essentiellement la fourberie de la figure réginale, qui use de diffamatio («mesparla») pour nuire à la réputation du héros.

Cette attaque injustifiée provoque la riposte de Lanval. Or, l'argument désormais avancé par lui n'est plus celui de sa fidélité vassalique au roi, mais celui de l'amour et surtout de la beauté de son amie faé, qu'il affirme largement supérieure à celle de la reine. Cette dernière se sent cette fois légitimement injuriée car sa beauté réginale est considérée comme indépassable. Il ne faudrait pourtant pas oublier que si Lanval s'emporte, c'est parce que la souveraine l'a provoqué en premier lieu. De surcroît, lorsqu'elle va se plaindre du chevalier auprès d'Arthur,

\footnotetext{
${ }^{14}$ Marie de France, «Lanval », dans Lais bretons (XII -XIII siècle) : Marie de France et ses contemporains, éd. et trad. Nathalie Koble et Mireille Séguy, Paris, Honoré Champion, coll. "Champion Classiques », 2011, p. 356, v. 275-286.

15 Rappelons que, d'après Nicole Gonthier, «cüarz » est à rattacher étymologiquement à cos, cous, "les testicules»; quant à " failliz », il provient du verbe latin fallere, "manquer à ». Le choix lexical de la reine est remarquable en ce qu'il opère un glissement, vers une figure masculine, de la polarisation sexuelle des insultes qui concerne traditionnellement les personnages féminins.
} 
elle ment « [e] dit que Lanval l'ad hunie : /De druërie la requist ${ }^{16} »$, écho manifeste au motif de la femme de Putiphar, qui confirme la duplicité du personnage ${ }^{17}$.

Réelle ou imaginaire, l'injure faite à la reine doit moins se lire comme la cause de la fragilisation de l'autorité royale que comme son symptôme. En somme, le ver est déjà dans le fruit et crée les conditions de possibilité de l'affront, qui n'est alors que la suite logique d'une chaîne de causalité débutée bien en amont. Dans le cas d'Aliscans, la faiblesse du souverain menace l'exercice du pouvoir royal; dans celui de Lanval, la malveillance et l'injustice du couple royal portent atteinte à la valeur conférée à la royauté ${ }^{18}$.

\section{De l'humiliation de la reine à l'honneur de la royauté : enjeux et modalités de la réparation de l'injure}

Sur le plan diégétique, l'outrage infligé à la reine revêt des conséquences non négligeables. Parce qu'il expose au grand jour les défaillances inhérentes au couple royal, il constitue le point culminant

\footnotetext{
${ }^{16}$ Marie de France, « Lanval », éd. cit., p. 358, v. 316-317.

${ }^{17}$ Le portrait de cette male reine redouble en fait celui d'un suzerain injuste à force de négligence. Trop prompt à croire le récit de son épouse sur parole, il prend ouvertement fait et cause pour elle : il accuse Lanval d'avoir doublement injurié la reine en requérant ses faveurs, puis en niant la prééminence de sa beauté ; de plus, il lui reproche de l'avoir lui-même déshonoré en tant que roi. Au tout début du récit, Arthur s'était déjà rendu coupable d'une injustice auprès de son fidèle vassal, en oubliant de le gratifier d'une femme et d'une terre lors de la distribution de dons aux seigneurs du royaume.

18 «Il y a valeur lorsqu'il y a reconnaissance d'une mission supérieure, d'une transcendance par rapport à l'ensemble du corps social, que cette mission ait un caractère religieux ou simplement politique et moral. Tandis que le pouvoir s'affirme unilatéralement, la valeur se reconnaît et suppose un consensus. ", Dominique Boutet, "Les chansons de geste et l'affermissement du pouvoir royal (1100-1250)», Annales. Économies, sociétés, civilisations, $37^{\mathrm{e}}$ année, $\mathrm{n}^{\circ} 1,1982$, p. 3-14, cit. p. 4 (nous soulignons). En l'occurrence, la valeur s'attache à l'institution monarchique en tant que régime politique ; le pouvoir concerne l'exercice de l'autorité par le couple royal. Ainsi la royauté peut-elle se caractériser, par exemple, par une haute valeur mais un pouvoir faible ou, inversement, par une basse valeur mais un pouvoir fort.
} 
d'une crise de l'autorité. Surtout, les insuffisances dégradant l'image des personnes en charge du pouvoir menacent, par analogie, d'affecter celle de la royauté en tant qu'institution. Au temps de la crise doit donc instamment succéder un temps de la résolution, qui pose la question de la réparation de l'offense et, corrélativement, de la restauration de l'honneur bafoué.

\section{Pour une restauration totale de l'honneur réginal : le chevalier et la reine}

Dans le roman arthurien, la résolution de l'avanie met généralement en scène un chevalier désigné ou auto-proclamé champion de la reine : le plus souvent, il efface l'infamie subie par cette dernière, en châtiant le ou les responsables. L'enlèvement de Guenièvre par Méléagant, après un défi lancé à Arthur dans Le Chevalier de la Charrette, constitue une véritable atteinte à l'intégrité physique de la figure réginale. Délivrant la souveraine de son ravisseur et la ramenant auprès de son époux, le chevalier Lancelot parachève la vengeance de l'affront en tranchant la tête de Méléagant.

Le Roman de la Rose de Jean Renart présente un schéma comparable, quoique très atypique dans ses motifs. La jeune Liénor, sœur de Guillaume de Dole, est promise à l'empereur Conrad. Le sénéchal de ce dernier est jaloux de Guillaume qui a les faveurs du monarque. Il décide donc d'entacher la réputation de Liénor en prétendant auprès du souverain qu'il l'a déflorée ${ }^{19}$. La spécificité de ce récit tient au fait que, dans une redistribution des rôles traditionnels, c'est la jeune femme elle-même qui entreprend de laver l'affront qu'elle a subi, et non son

\footnotetext{
${ }^{19}$ Certes, Liénor n'est pas encore impératrice à ce stade de l'œuvre, mais parce qu'elle a été promise solennellement à l'empereur, elle lui est symboliquement liée, quasiment au même titre que si elle était déjà son épouse. Dès lors, l'injure qui lui est faite joue de façon similaire à celle portée contre une reine officielle.
} 
frère Guillaume ou quelque autre champion masculin. Le diffamateur est finalement confondu au terme d'une série d'épreuves, propre à souligner toute l'habileté du personnage féminin. Reste alors à décider d'un châtiment pour le sénéchal, d'où l'intervention des barons du royaume pour conseiller Liénor, tout juste mariée à l'empereur :

En mi la requeste li distrent/que bien seüst ele une rien, /que l'empereres voloit bien/qu'ele feïst tot son voloir. / Hui més en porrai gë avoir, /fet ele, male volenté, /se vos m'avez dit verité, /que mis sires l'ait mis sor moi./Je ne voeil pa, ne ne le doi,/encor atrere en Alemaigne/que Dex et la gent ne s'en plaigne, /qu'il n'apartendroit pas a nos./Or m'en conselliez entre vos/quele justice on en puet fere, /sanz mort reçoivre et sanz deffere. $»^{20}$

Ce qui frappe ici, c'est la confiance absolue de Conrad en son épouse : il va jusqu'à lui laisser toute latitude quant à la sanction à réserver au félon. Le verdict extrêmement magnanime de Liénor, qui choisit d'épargner la vie du sénéchal, accroît nécessairement l'honneur de la nouvelle impératrice, comme le prouvent d'ailleurs les réactions des barons et du traître lui-même, lequel lui «ren[d] les grez et les merciz/de la bonté qu'el li ot fete ${ }^{21} »$. Par analogie, c'est surtout l'image de l'institution impériale, érigée en modèle de justice et de clémence, qui s'en trouve exaltée.

$\mathrm{Au}$ cours de ces entreprises de vengeance de l'offense, un personnage brille par son absence: le souverain lui-même ${ }^{22}$. Tenu à

\footnotetext{
${ }^{20}$ Jean Renart, Le Roman de la Rose ou de Guillaume de Dole, éd. et trad. Jean Dufournet, avec le texte édité par Félix Lecoy, Paris, Honoré Champion, coll. « Champion Classiques », 2008, p. 408, v. 5567-5581.

${ }^{21}$ Ibid., p. 412, v. 5619-5620.

${ }^{22}$ Dominique Boutet lit principalement, dans cette mise en retrait systématique du monarque, le résultat progressif de " [1'] écart entre les impératifs de la condition de roi et le goût de l'exploit de type chevaleresque »: en effet, «le roi, en risquant inconsidérément sa vie, met en péril le royaume, là où le chevalier ne met en péril que sa propre personne ». Voir Dominique Boutet, Charlemagne et Arthur, ou le roi
} 
l'écart de la réparation de l'injure, il laisse à d'autres le soin de restaurer l'honneur blessé, voire de le rehausser. Aussi le chevalier et, bien plus rarement, la reine trouvent-ils là matière à se distinguer. Toutefois, s'il leur concède de facto le terrain des aventures, le roi n'est pas pour autant remis en cause dans sa fonction. Au contraire, les actions chevaleresques et réginales décrivent une logique de soutien à la couronne. C'est que, dans ces romans, la valeur conférée à l'institution royale est toujours très haute : jamais Conrad ne voit son prestige diminuer, pas même après le supposé déshonneur de Liénor; quant aux récits arthuriens, ils sont marqués du sceau d'un idéal spécifique, celui d'une royauté « très haute en valeur, d'une royauté qui se définit uniquement comme une valeur ${ }^{23} »$. La fonction royale, dans ces univers romanesques, n'est par conséquent jamais vraiment menacée dans sa valeur. C'est davantage le pouvoir qui, bien qu'exercé par un roi debonaire, apparaît comme étant faible ${ }^{24}$. L'entreprise de réparation de l'offense faite à la reine revêt, dans ces conditions, un rôle d'affermissement du pouvoir et de réaffirmation de la valeur du statut royal. Dès lors, cet axe diégétique ne peut passer que par une restauration intégrale et éclatante, c'est-à-dire sans équivoque et publique, de l'honneur réginal.

Ces récits de résolution explicite et complète de l'affront dirigé contre une souveraine sont les plus fréquents. Reflétant une conception de la royauté investie d'une haute valeur et incarnée par un souverain

imaginaire, Paris, Honoré Champion, coll. « Nouvelle bibliothèque du Moyen Âge », 1992, p. 41-42.

${ }^{23} I d$., «Les chansons de geste et l'affermissement du pouvoir royal (1100-1250)», art. cit., p. 13.

${ }^{24}$ Encore cette faiblesse concerne-t-elle surtout le monde arthurien tel que le dépeint Chrétien de Troyes. Bien que fondée sur une très haute valeur, la royauté d'Arthur est simultanément caractérisée par un pouvoir faible. Voilà pourquoi le Chevalier Vermeil, dans Le Conte du Graal, et Méléagant, dans Le Chevalier de la Charrette, se permettent de le défier ouvertement et d'injurier la reine. L'autorité impériale exercée dans Le Roman de la Rose, elle, apparaît plus forte, seulement amoindrie sur le plan symbolique (et encore, provisoirement) par les manœuvres sournoises du sénéchal. 
debonaire, ils s'achèvent par l'indéniable victoire du personnage chargé de restaurer l'honneur bafoué (un chevalier ou la victime elle-même), donc par la valorisation de l'image de l'institution royale et, généralement, par la réprobation publique plus ou moins sévère de l'auteur de l'injure.

\section{Restaurer l'honneur de la reine? Le cas ambigu d'Aliscans}

À ce script narratif répond celui d'Aliscans, sensiblement différent. Pourtant, en exhibant l'humiliation de la reine Blanchefleur devant toute la cour, la chanson semble s'inscrire dans une trame tout à fait typique et poser le cadre nécessaire à la mise en scène d'une réparation totale et sans ambiguïé de l'avanie. La réaction de la souveraine, qui présente ses excuses à son frère, ne laisse dès lors pas de surprendre :

«Sire », dist ele, «ne sui mie marrie,/Ja n'i avrai honte ne vilenie. /Mes freres estes, mout en sui repentie. /Se j'ai dit chose dont m'aiez enhaie, /J'en souferrei, s'il vos plest, tel hachie/Que del paleis m'en irai despoillie,/Trestoute nue sanz chemise vestie, /Jusqu'au mostier Saint Vincent l'abaïe. »/Dont s'agenoille, a ses piez s'umelie, /Le pié li baise, s'a la jambe enbracie./Li quens Guillelmes l'en a amont drecie, /Enz la face l'a .IIII. foiz besie ${ }^{25}$.

Poussée par sa fille Aélis, qui a réprouvé l'égoïsme de sa mère lors de la requête de Guillaume, Blanchefleur se repent et, dans un étonnant renversement du scénario attendu, nie même avoir subi un préjudice de la part du comte d'Orange, comme le prouvent les tours négatifs « ne sui mie marrie » et « Ja n'i avrai honte ne vilenie ». Plus encore, l'épouse de Louis va jusqu'à s'humilier, dans une symbolique gestuelle très signifiante: elle adopte volontairement une posture de soumission en

${ }^{25}$ Aliscans, éd. cit., LXX, p. 248-250, v. 3383-3394. 
s'agenouillant devant son frère d'une part, et en lui baisant le pied d'autre part. Comment expliquer un tel choix diégétique?

Pour comprendre ce qui se joue dans cette séquence, il faut se pencher sur la représentation du roi dans Aliscans. À la différence de l'Arthur debonaire de Chrétien de Troyes, la faiblesse qui caractérise le Louis de la chanson de geste n'est pas uniquement d'ordre politique : comme examiné supra, il s'agit également d'une faiblesse morale qui, à force d'indolence et d'ingratitude, confine à l'injustice. Cristallisation des tensions entre un pouvoir royal veule et décevant dans son incarnation ludovicienne, et un pouvoir vassalique fort et juste dans sa personnification guillelmienne, la scène de Laon mène presque le pacte féodo-vassalique jusqu'au point de rupture. La couronne risque donc de perdre son plus puissant soutien.

Cette menace imminente permet d'éclairer l'attitude déroutante de Blanchefleur. Son geste de soumission face à son comte de frère, et ce alors même qu'elle est reine, suggère qu'elle accorde ici la préséance non pas à la hiérarchie féodale, comme on aurait pu le penser, mais à la hiérarchie lignagère. Partant, c'est moins en sa qualité de souveraine que de sœur qu'elle reconnaît avoir fauté envers son «frere » et «sire» Guillaume, et qu'elle fait amende honorable. Ce détour par le lien lignager est loin d'être fortuit: en déplaçant l'enjeu de la réconciliation du domaine politique au terrain familial, Blanchefleur atténue non seulement la gravité du crime de lèse-majesté perpétré par le comte d'Orange, mais préserve également son mari des éclaboussures de l'altercation. Tout se passe donc comme s'il s'agissait, pour contenir la crise, de minimiser la portée politique de l'injure en la réduisant à une simple querelle familiale ${ }^{26}$. D'ailleurs, contrairement au frère et à la sœur

\footnotetext{
${ }^{26}$ Dominique Boutet, Formes littéraires et conscience historique aux origines de la littérature française (1100-1250), Paris, PUF, 1999, p. 178.
} 
omniprésents, le roi se fait ici très discret, de l'instant où son épouse est outragée jusqu'à la clôture de l'incident. Dès lors que le voilà écarté de la résolution de l'affront, c'est Blanchefleur qui, par son attitude de soumission, endosse l'entière responsabilité des fautes commises par le couple royal.

Une telle stratégie de polarisation des défaillances sur la seule souveraine, alors même que les torts devraient être partagés, présente un intérêt manifeste : la souillure étant nettement circonscrite à la personne de la reine, le roi, bien que répréhensible lui aussi, sauve officiellement la face $^{27}$. Nul n'est dupe, bien sûr, et certainement pas l'auditeur-lecteur, mais du moins l'image de l'institution royale est-elle épargnée grâce à cette nette dissociation entre figure régalienne et figure réginale. C'est dire si, en dépit des apparences, l'idéologie de la chanson d'Aliscans est marquée par une valeur fondamentalement haute de la royauté : les auteurs ont beau rudoyer Louis aux épaules trop maigres pour sa lourde tâche, ils tiennent à réaffirmer, dans l'après de l'injure, la nécessité de se montrer fidèle au principe monarchique ${ }^{28}$. Il faut savoir jusqu'où aller trop loin...

Que la démarche de Blanchefleur soit, du point de vue de la diégèse, dictée par le calcul politique, ou simplement par la peur du courroux fraternel, ne change rien à l'affaire : les conséquences restent identiques et sont loin d'être négligeables. Sur le plan privé, une telle ligne narrative conduit, à travers la réconciliation du frère et de la sœur, à

\footnotetext{
${ }^{27}$ Significativement, jamais le monarque ne présente d'excuses formelles à son beaufrère. Il ne sort de son mutisme et ne reprend part à l'action, une fois l'entente revenue, que pour se camper dans une posture régalienne topique d'hôte parfait incarnant l'idéal de largesse : «Li rois commande la table soit drecie, /Cele qui est a fin or entaillie », Aliscans, éd. cit., LXX, p. 250, v. 3400-3401.

${ }^{28}$ Sur ce paradoxe du «plaidoyer a contrario en faveur de la royauté », qui consiste à incarner la fonction royale dans une figure négative pour souligner, par contraste, la très haute valeur conférée à l'institution royale, voir Dominique Boutet, "Les chansons de geste et l'affermissement du pouvoir royal (1100-1250) », art. cit., p. 7.
} 
la restauration de la cohésion et de l'harmonie au sein du lignage. Sur le plan politique, le rétablissement de la concorde lignagère induit, de manière concomitante, un renoncement tacite au projet de rupture du pacte vassalique de la part de Guillaume. De facto, ce dernier se replace sous le pouvoir régalien de Louis, dont il reconnaît l'autorité et la suprématie. Désamorcer la crise familiale revient donc à désamorcer la crise politique; restaurer le lien lignager revient à protéger le lien féodo-vassalique. Ainsi, le retour à l'ordre est garanti, dans une résolution pacifique de l'injure. Certes, l'honneur réginal n'est officiellement pas restauré, dans la mesure où il ne fait pas l'objet d'une réparation éclatante et sans équivoque. Mais l'acte d'humiliation repentante de Blanchefleur lui permet, du moins, de recouvrer son honneur lignager et de s'offrir la possibilité d'un rachat $^{29}$. Le personnage parvient ainsi à un subtil équilibre : sauvegarder l'image de l'institution royale, tout en échappant de justesse au rôle de bouc émissaire.

Ambiguë car partielle, la résolution de l'outrage fait à la reine dans Aliscans est intrinsèquement liée à la conception de la fonction royale reflétée par la chanson. Incarnée par un roi incontestablement défaillant, mais dotée d'une haute valeur, la royauté ne saurait finalement perdre la face $^{30}$. La canalisation de la souillure sur la seule personne de la reine permet alors, en endiguant la contagion, de préserver officiellement l'image du roi et, à travers elle, celle du statut royal. Blanchefleur agit ici à la fois comme un intermédiaire entre son frère-vassal et son époux-roi, et comme un paratonnerre pour le roi et la royauté.

\footnotetext{
${ }^{29}$ Signe que ses fautes ont été pardonnées, la souveraine est en effet récompensée de son humilité par sa réintégration au sein du lignage, bien visible à travers la symbolique gestuelle: dans un témoignage d'affection qui en dit long, le comte d'Orange a en « amont drecie » sa sœur et « [e]nz la face l'a .IIII. foiz besie », scellant ainsi leur réconciliation, et restituant du même coup à Blanchefleur la place qui lui est due au sein du lignage (Aliscans, éd. cit., LXX, p. 250, v. 3393-3394).

${ }^{30}$ Dominique Boutet, Charlemagne et Arthur, ou le roi imaginaire, op. cit., p. 418.
} 


\section{Condamner la reine : au-delà de l'injure, la critique du monde féodal}

Le lai de Lanval, dont la seconde moitié est dominée par le procès que le roi fait instruire contre son vassal ${ }^{31}$, se démarque par une résolution assez surprenante de l'affront : d'une part, grâce à l'intervention de l'être faé à la cour d'Arthur, le héros est acquitté ; d'autre part, le récit se clôt sur un schéma de type "morganien », avec le départ pour Avalon du couple principal formé par le chevalier et son amie ${ }^{32}$. Si ce dénouement signe bien un retour à l'ordre, celui-ci se révèle pour le moins problématique. Innocenter Lanval revient en effet à donner tort au couple royal qui l'avait mis en accusation, donc à laisser officiellement impunie l'injure faite à la reine. Surtout, le choix manifeste du protagoniste en faveur de l'Autre Monde résonne corrélativement comme un net désaveu du royaume arthurien. Ainsi, là où la résolution de l'outrage se fait d'ordinaire dans la reconnaissance de la suprématie royale, elle est marquée ici par le discrédit jeté sur la royauté incarnée par le légendaire souverain de Logres.

Il faut dire qu'Arthur semble dans ce lai particulièrement indigne de sa charge : de sa négligence initiale lorsqu'il oublie de récompenser Lanval, à son incapacité à «faire régner le droit et la justice ${ }^{33}$ », en passant par son aveuglement face aux manœuvres de son épouse, ses défaillances sont légion et entament considérablement la valeur de sa

\footnotetext{
${ }^{31}$ Celui-ci est accusé d'avoir attenté à la pudeur de la reine en requérant ses faveurs, puis à sa beauté en se vantant d'avoir une amie plus belle qu'elle.

${ }^{32} \mathrm{Ce}$ schéma narratif met en scène un être surnaturel qui s'éprend d'un humain et l'entraîne dans l'Autre Monde. Voir Laurence Harf-Lancner, Les Fées au Moyen Âge. Morgane et Mélusine ou la naissance des fées, Paris, Honoré Champion, 1984, p. 10.

${ }^{33}$ Christopher Lucken, «L'aventure d'une autre loi : justice féérique dans le lai de Lanval », dans Les Possibles de la narration dans les Lais de Marie de France, Actes de la Journée d'étude organisée le 8 janvier 2019 à l'Université Toulouse II-Jean Jaurès, dir. Éléonore Andrieu, p. 23-43, cit. p. 37 [URL : http://www.univ-paris3.fr /publications-de-la-silc-section-francaise--393070.kjsp?RH=1329834238527].
} 
royauté. Dès lors que le monde féodal de l'île de Bretagne apparaît injuste, corrompu, décevant, l'Autre Monde insulaire d'Avalon s'érige, lui, en lieu de «compensation ou de consolation pour des vassaux maltraités par leur seigneur [...] qui peuvent trouver une sorte de revanche dans l'imaginaire ou le merveilleux ${ }^{34} »$.

Cette concurrence entre monde féodal et monde merveilleux se cristallise dans la rivalité opposant la reine à la fée. Si la première tente initialement de nier l'existence de la seconde en prétendant que nulle ne saurait l'éclipser en beauté, c'est bien l'être surnaturel qui surclasse finalement la souveraine. Son éblouissante apparition à la cour d'Arthur suffit à disqualifier sa rivale. Par sa seule présence, la fée donne raison aux propos tenus par Lanval sur sa beauté et discrédite ainsi les allégations de la male reine. Poussant son avantage, elle glisse cette phrase à Arthur qui porte le coup de grâce à son ennemie : «[...] ceo saches tu /Que la reïne ad tort eü : /Unkes nul jur ne la requist ${ }^{35}{ }$. Le double renversement à l'œuvre ici est pour le moins éloquent : d'une part, l'accusation d'atteinte à la pudeur est dénoncée pour ce qu'elle est, à savoir une injure imaginaire; d'autre part, d'accusatrice qu'elle était, la souveraine passe désormais en position d'accusée.

À l'instar de Blanchefleur dans Aliscans, l'épouse d'Arthur concentre donc sur elle tous les torts. Mais le lai se fait davantage explicite que la chanson de geste, en désignant nommément ce personnage comme coupable. Plus encore, le dénouement ne lui laisse aucune chance de se défendre: une fois prononcé l'acquittement de Lanval, le texte s'achève en effet rapidement, sans que quiconque prenne

\footnotetext{
${ }^{34}$ Hélène Débax, «Fidélité, vassalité, féodalité dans les Lais de Marie de France », dans ibid., p. 13-22, cit. p. 22. Significativement, c'est la fée, être surnaturel, qui apporte les preuves de l'innocence du héros et permet le triomphe de la justice. On comprend donc mieux que Lanval ait choisi de s'exiler en Avalon, le royaume arthurien n'étant pas suffisamment vertueux pour retenir un chevalier de sa valeur...

${ }^{35}$ Marie de France, « Lanval », éd. cit., p. 384, v. 619-621.
} 
la parole pour répondre à l'accusation de l'être surnaturel contre la reine. Déniant à l'auditeur-lecteur le moindre aperçu du futur, la conteuse clôt son récit sur un silence qui, s'il laisse en suspens la question de la destinée des amours de Lanval et de son amie ${ }^{36}$, préserve surtout le mystère quant au devenir de la souveraine. L'accusation de la fée constitue donc la dernière parole énoncée au sujet de la figure réginale. Elle sonne alors à la fois comme une vérité assourdissante et une condamnation sans appel. Ne faisant l'objet d'aucune tentative de réparation, l'honneur de la suzeraine reste ainsi souillé pour une durée potentiellement illimitée. Plus encore, n'exprimant aucun remords pour sa perfidie, la reine se prive de toute possibilité de rachat.

Pendant logique à cette polarisation des fautes sur la personne de la souveraine, la responsabilité d'Arthur est tue, alors même que les torts envers Lanval sont clairement partagés. Cette dissociation entre les figures régalienne et réginale dans les derniers vers semble résulter, comme pour Aliscans, d'une stratégie narrative visant à ménager un tant soit peu l'image publique du roi, fortement égratignée au sein du texte.

La différence majeure entre les deux œuvres réside en fait dans l'idéologie propre à chacune : ici, la valeur conférée à la royauté est basse, ce qui oriente toute la lecture du dénouement du lai et l'interprétation du rôle dévolu à la souveraine. S'il n'est pas remis en cause à proprement parler, du moins le régime monarchique se voit-il sondé et interrogé dans ses déficiences et ses possibles dérives. Pour autant, pointer les défaillances de la royauté n'implique pas de dénigrer complètement le roi. Bien que loin d'être exempt de tout reproche, celuici ne saurait être entièrement désavoué ; le maintien de l'ordre en dépend.

\footnotetext{
${ }^{36}$ Jean-Marie Fritz, «Le clos et l'ouvert dans les Lais de Marie de France », dans Les Possibles de la narration dans les Lais de Marie de France, op. cit., p. 45-52, voir p. 45,49 et 51 .
} 
La malveillance de la reine apparaît dès lors comme un prétexte tout trouvé pour justifier, à défaut d'excuser, les manquements du monarque, au fond davantage victime de la mauvaise influence de son épouse que véritablement malfaisant ${ }^{37}$.

Aboutissant non seulement à une absence totale de restauration de l'honneur réginal, mais aussi à une condamnation sans appel de l'épouse d'Arthur, la résolution de l'injure dans Lanval fait de la souveraine le bouc émissaire d'une royauté défaillante à tous points de vue et à laquelle n'est conférée qu'une basse valeur. Si cette concentration des torts sur la male reine ne réhabilite nullement son époux, elle fait malgré tout figure de mal nécessaire : en octroyant des circonstances atténuantes au roi, elle évite au lai de basculer dans une opposition complète et définitive à la royauté.

Véritable baromètre des forces et des faiblesses du pouvoir royal, l'injure faite à la reine dans la littérature du Moyen Âge central ne saurait être pensée séparément de l'image publique du roi et, par analogie, de celle de la royauté. L'après de l'offense appelle donc nécessairement une résolution qui passe par un retour à l'ordre, mais emprunte des chemins différents en fonction de la valeur conférée à l'institution royale : plus celle-ci est haute et incarnée par un souverain debonaire, plus la réparation de l'injure à la reine se fait éclatante et la restauration de l'honneur sans équivoque; à l'inverse, plus cette valeur est basse et

\footnotetext{
${ }^{37}$ Cette lecture nous semble cohérente avec l'hypothèse, maintes fois avancée, selon laquelle Henri II Plantagenêt serait le dédicataire des Lais. Le choix du légendaire Arthur par Marie de France n'est pas anodin : on sait combien Henri II s'est employé, pour des raisons politiques, à lier son règne à l'ascendance prestigieuse que représentait la figure mythique du roi de Logres. Dans ce lai empreint de didactisme, le personnage d'Arthur agit sans doute comme un contre-modèle destiné à mettre en garde le souverain anglais contre les dérives du mauvais gouvernement. Néanmoins, n'est-il pas légitime de supposer que la forte identification d'Henri II à Arthur s'accommoderait mal d'une réprobation sans nuance du modèle?
} 
incarnée par un suzerain injuste, plus la réparation de l'outrage et la restauration de l'honneur réginal sont ambiguës, voire inexistantes. C'est que la relation asymétrique qui unit les époux royaux dessine, dans l'après de l'injure, un pacte de solidarité à géométrie variable. Là où le personnage régalien peut être dissocié de la souveraine au gré des circonstances et des intérêts de la couronne, la figure réginale - tour à tour instrument d'affermissement du pouvoir et de revalorisation du statut royal, intermédiaire entre le vassal et le souverain, pièce d'échecs pouvant être sacrifiée si besoin - fait, elle, office d'outil narratif et diégétique, mis inconditionnellement au service de la préservation de l'honneur du roi et de celui de la royauté. 
